\title{
STABILITY ASSESSMENT AS A CRITERION OF STABILIZATION OF THE MOVEMENT TRAJECTORY OF MOBILE CRANE WORKING ELEMENTS
}

\author{
W. KACALAK and Z. BUDNIAK ${ }^{*}$ and M. MAJEWSKI \\ Faculty of Mechanical Engineering, Koszalin University of Technology \\ Raclawicka 15-17, 75-620 Koszalin, POLAND \\ E-mails: wojciech.kacalak@tu.koszalin.pl; zbigniew.budniak@tu.koszalin.pl \\ maciej.majewski@tu.koszalin.pl
}

\begin{abstract}
The article presents a stability assessment method of the mobile crane handling system based on the safety indicator values that were accepted as the trajectory optimization criterion. With the use of the mathematical model built and the model built in the integrated CAD/CAE environment, analyses were conducted of the displacements of the mass centre of the crane system, reactions of the outrigger system, stabilizing and overturning torques that act on the crane as well as the safety indicator values for the given movement trajectories of the crane working elements.
\end{abstract}

Key words: mobile crane, stability of a crane, overturning torque, lifting capacity, tip-over axis.

\section{Introduction}

Carrying large loads with the aid of truck mobile cranes may in certain conditions lead to a stability loss [1-10]. The value of the moment required to maintain balance in relation to the tip-over axis $[2,8$, $11,12]$ may constitute the measure of the risk of the crane tipping over. Loading with the moment from the mass of the crane elements and the loads is additionally summed up with the moments that originate from inertia forces (caused by the movement of the cargo and its parts) and from the load with wind [2, 13-15]. The overturning torque $M w$ is counteracted by the stabilizing torque $M u$ with an opposite direction that is dependent on the mass and the location of the mass centre of the crane elements (Fig.1).

According to international [16] and Polish [17, 18] standards, it is accepted that the crane is stable if at any position of the boom loaded with lifting capacity with an adequate extension, the stabilizing torque $M u$ is greater than the overturning torque $M w$ by the value of $\Delta M$.

$$
\Delta M=M_{u}-M_{w}>0
$$

where

$$
\begin{aligned}
& \Delta M=\min \in\left(\Delta M_{i}\right), \quad \Delta M_{i}=M u_{i}-M w_{i}, \\
& M u_{i}=\sum_{j=1}^{n} G_{j} \cdot d_{i_{j}}, \quad M w_{i}=\sum_{j=n+1}^{m} G_{j} \cdot d_{i_{j}}, \\
& d_{l_{j}}=z_{j}-z_{S_{1}}, \quad d_{2_{j}}=x_{S 2}-x_{j}, \quad d_{3_{j}}=z_{S_{4}}-z_{j}, \quad d_{4_{j}}=x_{j}-x_{S_{1}}
\end{aligned}
$$

\footnotetext{
${ }^{*}$ To whom correspondence should be addressed
} 
$i=1 \div 4$ - number of the tip-over axis; $n$ - number of the elements of the crane system, whose weight vector $G_{i}$ in the time of cargo transport, in a projection on the horizontal plane $O x z$ is located inside the tip-over contour that is limited by axes: $k_{1}, k_{2}, k_{3}$ and $k_{4} ; m$ - number of all the crane system elements; $d i_{\mathrm{j}}$ - distance of the gravity centre of the element $j$ from the tip-over axis $i$ in the projection on the horizontal plane.

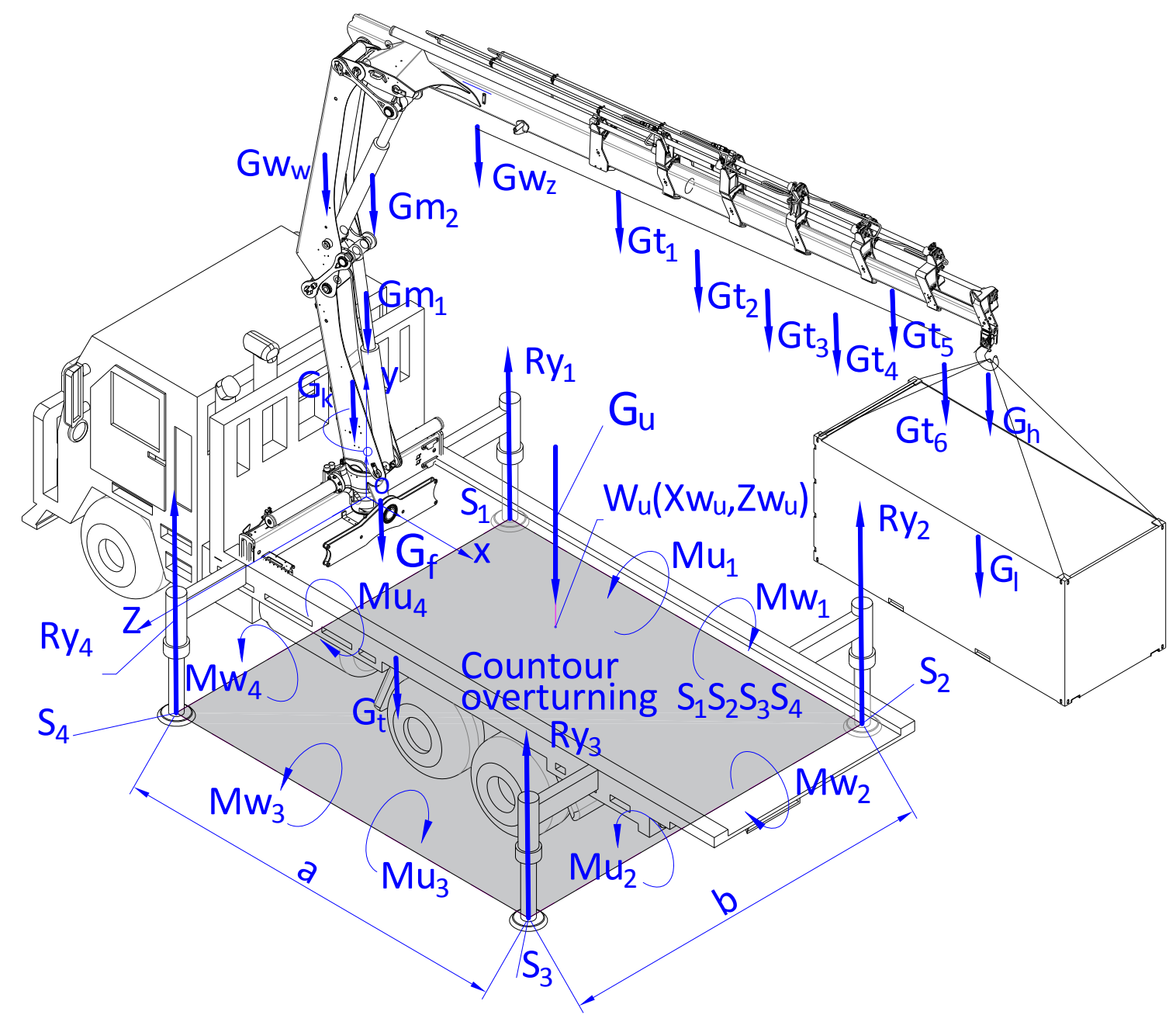

Fig.1. Diagram of forces and torques that act on the crane outrigger system: where: $G u$ - total weight of the crane system; $G f$ - weight of the truck including the outrigger system; $G b$-crane base weight; $G k-$ weight of the slewing column; $G w_{w}$ - weight of the inner arm, $G w_{z}$ - weight of the outer arm; $G m_{1}$, $G m_{2}$ - weights of hydraulic cylinders; $G t_{1}, G t_{2}, . ., G t_{6}$ - weights of the arms of the six-member crane boom; $G h$ - hook weight, $G l$ - cargo weight; $R y_{1}, R y_{2}, R y_{3}, R y_{4}$ - vertical reactions of the base; $a$ and $b$ - spacing of the crane outriggers.

The following may also constitute the measure of the crane stability:

- the value of the pressure on the base of the least loaded crane support and the value of the changes of this force in time $[2,19,20]$;

- the location of the symmetric mass centre of the handling system of the crane in relation to the support points $[2,3]$. The system is stable if, in the projection on the horizontal plane, the mass centre is located inside the quadrangle that is established by the support points of the crane outrigger system; 
- safety indicator $W b$. The authors presented a new effectiveness assessment method of the handling assignment that permits the determination of the value of the safety indicator $W b$ as a criterion of the stability of the crane system. The indicator $W b$ accepts values from 0 to 1 . The value of the indicator of $W b=0$ constitutes the lower limit of safe operation.

The paper presents a stability assessment of the mobile crane system based on the methodology developed with the use of a simulation model that was built in the integrated CAD/CAE environment. The model proposed consists of the main crane assemblies coupled together: the truck with outrigger system and the base, the slewing column, the inner and outer arms, the six-member telescopic boom, the hook with lifting sling and the transported load. In the modelling of the crane system, the masses of the majority of the equipment and the assemblies that load the system were taken into account.

The following changes were presented as the results of simulation testing: the location of the mass centre of the crane system, the reaction of the outrigger system, stabilizing and an overturning torques that act on the crane and the values of the safety indicator depending of the location of the working elements of the machine.

\section{Methodology of the assessment of the stability of the crane handling system}

The methodology as presented in Fig.2 was used in simulation testing to assess the stability of the mobile crane handling system. The simulation model built with the use of the integrated CAD/CAE system makes it possible to assess the stability of a crane system, using the HIAB XS 111 crane as an example, with the proposed interaction and control system [20,21].

The following are the basic elements of the method implemented:

- parametric modelling of the elements and the entire crane system in the CAD system for the defined configuration;

- determination of the system stability conditions, i.e., equations that constitute a mathematical model to calculate the following: the trajectory of the mass centres of the elements of the crane system, the reaction of the base on the crane outrigger system, the stabilizing torque $M_{u}$ and the overturning torque $M_{w}$ as well as the safety indicator

- building of a kinematic model of the crane and carrying out simulation testing in the integrated $\mathrm{CAD} / \mathrm{CAE}$ system;

- an analysis of the kinematic and dynamic quantities of the crane system during handling in connection with maintaining constant balance (stability);

- an optimization of the trajectories of the displacements of the working systems of the crane for specific assignments taking into consideration the movement safety indicator considering the limiting conditions. by knowing the value of the safety indicator during working movements, it is possible to conduct an assessment of the risk of the loss of the crane's stability and to select the optimal displacement trajectory. parametric modelling of the elements and the entire crane system in the CAD system for the defined configuration;

- determination of the system stability conditions, i.e., equations that constitute a mathematical model to calculate the following: the trajectory of the mass centres of the elements of the crane system, the reaction of the base on the crane outrigger system, the stabilizing torque $M_{u}$ and the overturning torque $M_{w}$ as well as the safety indicator);

- building of a kinematic model of the crane and carrying out simulation testing in the integrated CAD/CAE system;

- an analysis of the kinematic and dynamic quantities of the crane system during handling in connection with maintaining constant balance (stability);

- an optimization of the trajectories of the displacements of the working systems of the crane for specific assignments taking into consideration the movement safety indicator considering the limiting conditions. By knowing the value of the safety indicator during working movements, it is possible to conduct an assessment of the risk of the loss of the crane's stability and to select the optimal displacement trajectory. 
Integrated CAD - SolidWorks software as well as the module for computations and engineering analyses: CAE - SolidWorks Motion was used for the purpose of the modelling and numerical tests of the crane handling system.

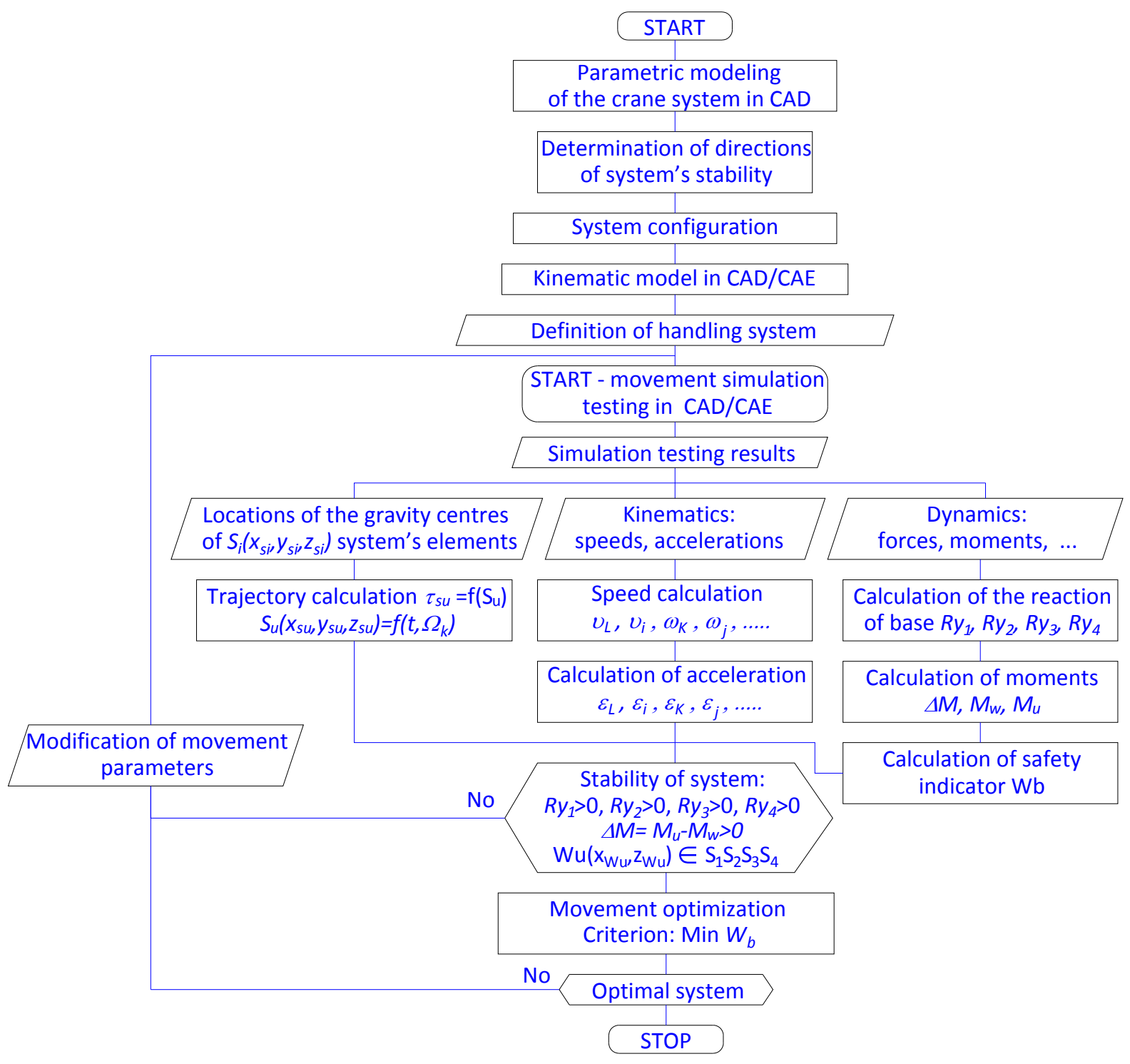

Fig.2. Block diagram of computer aided assessment of the stability of the crane handling system.

\section{Model of handling crane}

The assemblies of the truck crane type HDS HIAB XS (Cargotec Poland) in relation to which the voice control system was proposed - [21,22] include the design of all the main parts and sub-assemblies as well as other important elements of the construction $[3,19]$ shown in Fig.3.

The model of the support system is composed of the following crane assemblies that are coupled together: the truck frame, the outrigger system placed in the crane base frame connected with the frame of the truck chassis, the crane base, the slewing column, the inner and outer boom with the installed sixmember telescopic boom and the hook including lifting slings loaded with the transported cargo. 
All the geometric quantities as well as all the properties of the constructional elements used of the constructional models were entered to the file of the programme in a parametric manner. This manner allows for a complete control of changes to the geometric dimensions of the crane elements. The possibility to select any reciprocal position of the main crane assemblies with a selected accuracy is important as well.

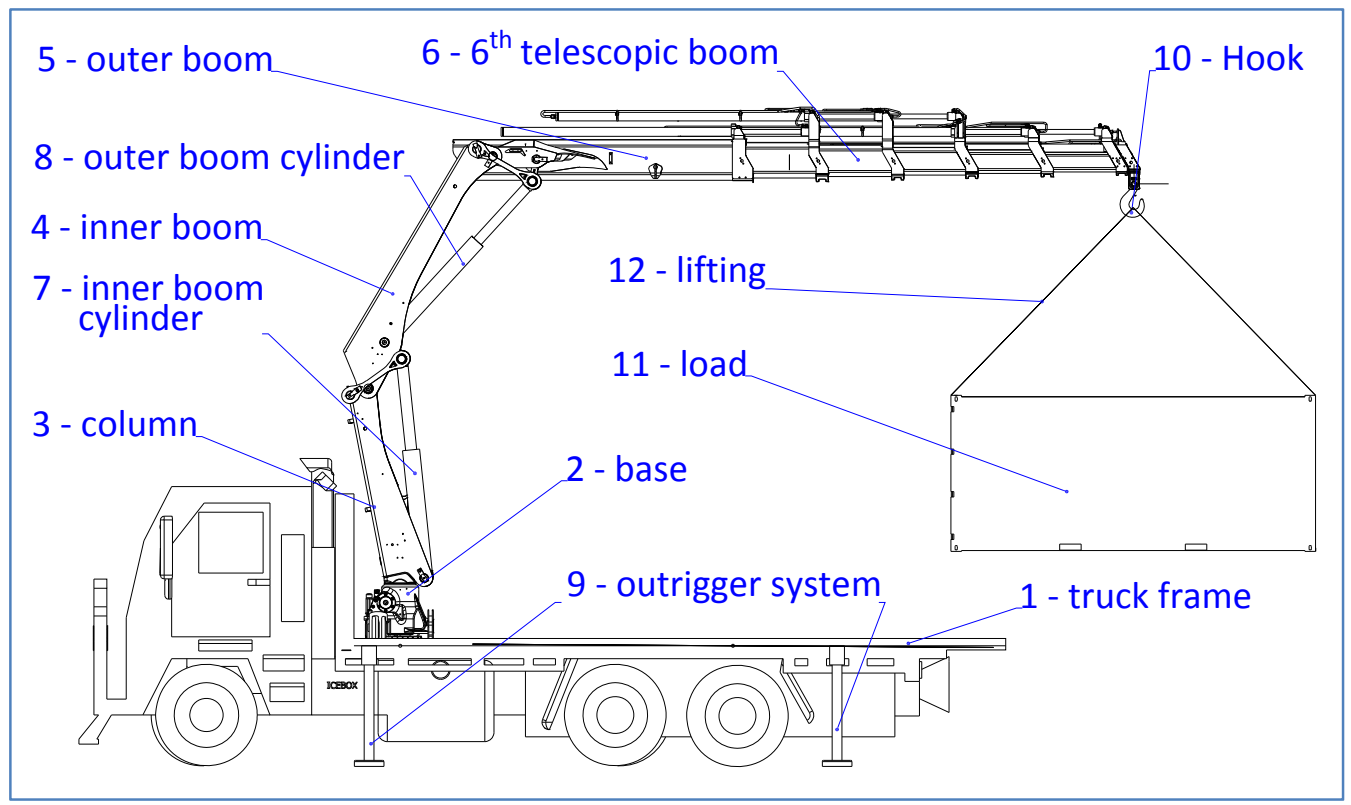

Fig.3. Handling system of mobile crane type HDS HIAB XS111.

The masses of the elements of the equipment and the assemblies that load the system $[3,19]$ were taken into account in the modelling of the crane. A change to the configuration of the crane system is connected with its working movements. An analytical description of the configuration of the crane kinematic system involves strenuous conversions of vector-matrix equations [7] until explicit dependences have been obtained that determine the variable angular and linear quantities. Knowledge of these dependences is very desirable. It needs to be emphasized, however, that it is very difficult to obtain explicit dependences for the crane handling system. The integrated CAD/CAE system was therefore used to determine vectors that specify the configuration of the crane system.

\section{Kinematic model of the handling system}

In simulation testing, a kinematic model was used of the mobile crane handling system with four degrees of freedom. Considering a large number of elements and their construction characteristics $[3,23,24]$, a number of necessary simplifications were used in the kinematic model (Fig.4):

- solid models of the important elements of the crane system were replaced with axis models (crane column $k$, outer arm $w_{w}$, inner arm $w_{z}$, telescopic boom $t$, hook $h$ and lifting sling $z$ ) located on the $\pi$ plane;

- the six-member telescopic boom with six degrees of freedom [3,24] including arms $t_{1}, t_{2}, t_{3}, t_{4}, t_{5}$ i $t_{6}$ was replaced with a single boom $t$. Owing to this simplification, it was possible to obtain a kinematic model with four degrees of freedom instead of nine degrees;

- mass, whose centre of gravity coincides with the gravity centre of the solid model, was ascribed to the individual elements of the system;

- the geometric dimensions of the elements of the rigging and solid models of the crane are identical.

In order to determine dependences between the configuration coordinates $\left(\varepsilon, \varepsilon_{b}, \varepsilon_{e}, \alpha, \alpha_{b}, \alpha_{e}, \beta, \beta_{b}\right.$, $\beta_{e}$, where: $b$ and $e$-indices for the initial and end positions) and the base coordinates of the location of the 
cargo, temporary 3D bonds were introduced into the simulation model, which determine the location of the handling system and its elements. In the model developed, drives were defined that perform the rotary motion of the crane column with velocity $\dot{\varepsilon}$ and linear drives that force the rotary motion of the inner and outer arms with velocities $\dot{\alpha}$ and $\dot{\beta}$ as well as sliding out of the six-member telescopic boom with velocity $\dot{\delta} t$.

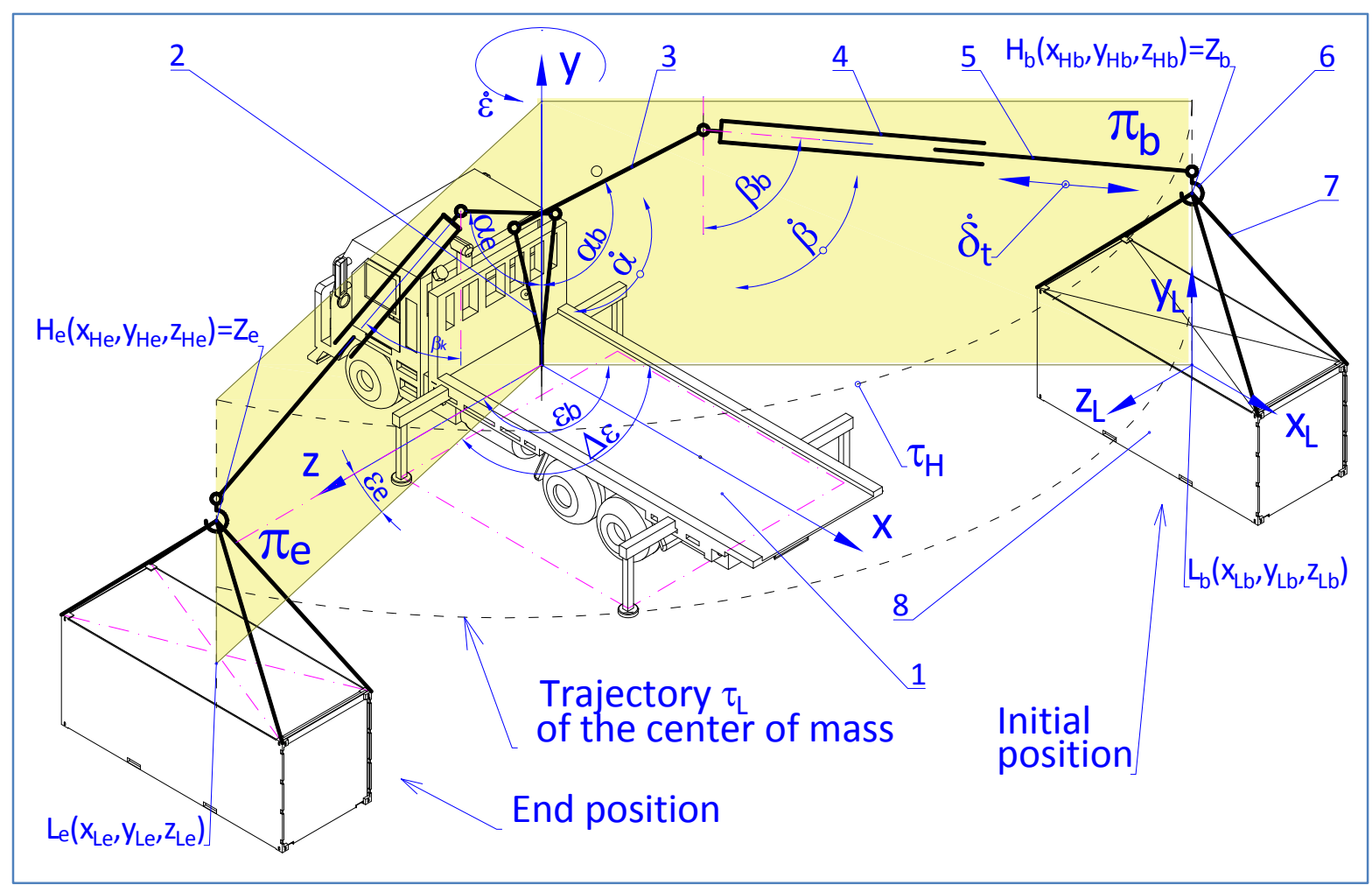

Fig.4. Simplified kinematic model of the crane handling system, where: 1 - truck including outrigger system, 2 - slewing column, 3 - inner arm, 4 - outer arm, 5 - telescopic boom, 6 - hook, 7 - lifting slings, 8 - cargo, $\tau_{L}$ - trajectory of cargo gravity centre, $\tau_{H}$ - trajectory of point $H=Z$ (hang point: lifting slings and hook).

\section{Safety indicator of the crane handling cycle}

The article presents a new method to assess the effectiveness of the handling assignment that permits a determination of the value of the safety indicator $W b$ as the stability criterion of the crane system.

The stability indicator $W b$ was defined as follows

$$
W b=\min \in\left\{\frac{\min \left(R y_{i}\right)_{t}}{G_{u} \cdot k_{1} \cdot\left(1-k_{2}\right)}-\frac{k_{2}}{1-k_{2}}\right\}_{t}
$$

where

$$
t=t_{e}-t_{b}, \quad t=\sum \Delta t_{j},
$$

$i=1-4$ - number of the outrigger, $j$ - number of the elementary fragment of the trajectory; $\min \left(R y_{i}\right), \mathrm{kN}$ the smallest of the vertical reactions of the base on the outrigger $i ; G u, \mathrm{kN}$ - total weight of the crane system, $k_{1}$ - index of the maximum load of the crane outrigger; $R y_{\max }=G u \cdot k_{1}$, where: $k_{1} \leq 0.25$ - for a crane with four outriggers; $k_{2}$ - index that determines the minimum load of the crane outrigger, $R y_{\min }=G u \cdot k_{2} ; t, s$ - time 
of the working cycle of the handling assignment; $t_{b}, s$ - start of the crane working cycle; $t_{e}, s$ - end of the crane working cycle.

In order to guarantee the stability of the crane system, the value of the indicator $W b$ should be greater than zero when $\min \left(R y_{i}\right)>k_{1} \cdot k_{2}$. The value of the indicator $k_{2}$ is determined considering safety on the level that depends on the crane working conditions. It was accepted that the value of this index takes into account the wind speed as well as the velocities, accelerations and pulls in the crane kinematic pairs. Pulls may be the result of the cargo frozen to the ground being torn off, the cargo being broken off, sudden breaking, hitting an obstacle, etc.

From the perspective of the general principles of the safe operation of the crane, it is accepted that the value of the index $k_{2}$ is proportional to the speed of the wind and crane elements as well as accelerations and pulls that occur in the kinematic pairs of lever devices. Provision of the value of the index $k_{2}$ is one of the several alternative methods to determine the safety stock of the crane operation.

The geometric interpretation of the index $W b$ and the stability evaluation of the handling assignment were conducted a mobile crane of the HDS HIAB XS111 type. The configuration of the movement of the working mechanisms of the crane during the execution of the three variants of the handling assignment is presented in Tab.1, where the denotations of the location parameters were accepted according to Fig.4. The cargo located in position $A$ was to be transported and positioned in location $B$ (Fig.5).

Table 1. Parameters of sequential movements for three variants of handling assignment.

\begin{tabular}{|c|c|c|c|}
\hline $\begin{array}{c}\text { Variant of cargo } \\
\text { displacements }\end{array}$ & I & II & III \\
\hline 1 & $\Delta \beta=7.2$ & $\Delta \beta=7.2$ & $\Delta \beta=7.2$ \\
\hline 2 & $\Delta \varepsilon=-189^{\circ}$ & $\Delta \delta t=-1.5 \mathrm{~m}$ & $\Delta \delta t=2.1 \mathrm{~m}$ \\
\hline 4 & $\Delta \delta t=2.1 \mathrm{~m}$ & $\Delta \varepsilon=-189^{\circ}$ & $\Delta \varepsilon=-189^{\circ}$ \\
\hline 4 & $\Delta \beta=-5.2^{\circ}$ & $\Delta \delta t=3.6 \mathrm{~m}$ & $\Delta \beta=-5.2^{\circ}$ \\
\hline 5 & - & $\Delta \beta=-5.2^{\circ}$ & - \\
\hline
\end{tabular}

The following assumptions were accepted for the needs of an assessment of the value of the safety indicator $W b$ in simulation testing:

- the crane is positioned on a stable horizontal base (inclination up to $1 \%$ );

- the rotation angle of the crane column was measured from the plane $O z y$ and its range $\varepsilon=0 \div 360^{\circ}$ was determined; the lifting angle of the outer boom, measured vertically, changed in the range of $\alpha=34.13^{\circ} \div 185.16^{\circ}$; the value of the angle of rotation of the inner arm was $\beta=9.9^{\circ} \div 182.5^{\circ}$;

- in the simulation testing, the following propeller speeds were accepted: $\dot{\varepsilon}=18 \mathrm{deg} / \mathrm{s}, \dot{\alpha}=2.5 \mathrm{deg} / \mathrm{s}, \dot{\beta}=5$ $\mathrm{deg} / \mathrm{s}, \dot{\delta} t=0.3 \mathrm{~m} / \mathrm{s}$

- the value of the extension of the telescopic boom was in the range of $\delta t=0 \div 11.85 \mathrm{~m}$;

- the value of the safety indicator was $k_{1}=0.25$. This means that in the most favourable position of the centre of the mass $W u\left(x_{W u}, z_{W u}\right)$ of the crane system, in a projection on the horizontal plane, all the vertical reactions in the outriggers are identical and they constitute $25 \%$ of the total load $G u$;

- the value of the safety indicator $k_{2}=0.05$;

- it was accepted in the simulation testing that the crane is not subject to the wind pressure force (the wind speed is smaller than $v_{w}<8.3 \mathrm{~m} / \mathrm{s}$ and it is neglected);

- the working movements of the crane are smoothly controlled, hence it was accepted that inertia forces can be neglected;

- the values of the masses of the crane elements and the locations of their gravity centres were taken into account in modelling; 
- the mass of the cargo carried is $m_{l}=560 \mathrm{~kg}$;

- in the initial position, the configuration coordinates of the individual working mechanisms are as follows: $\varepsilon_{p}=135^{\circ}, \alpha_{p}=112^{\circ}, \beta_{p}=139.8^{\circ}, \delta t_{p}=2.4 \mathrm{~m}$.

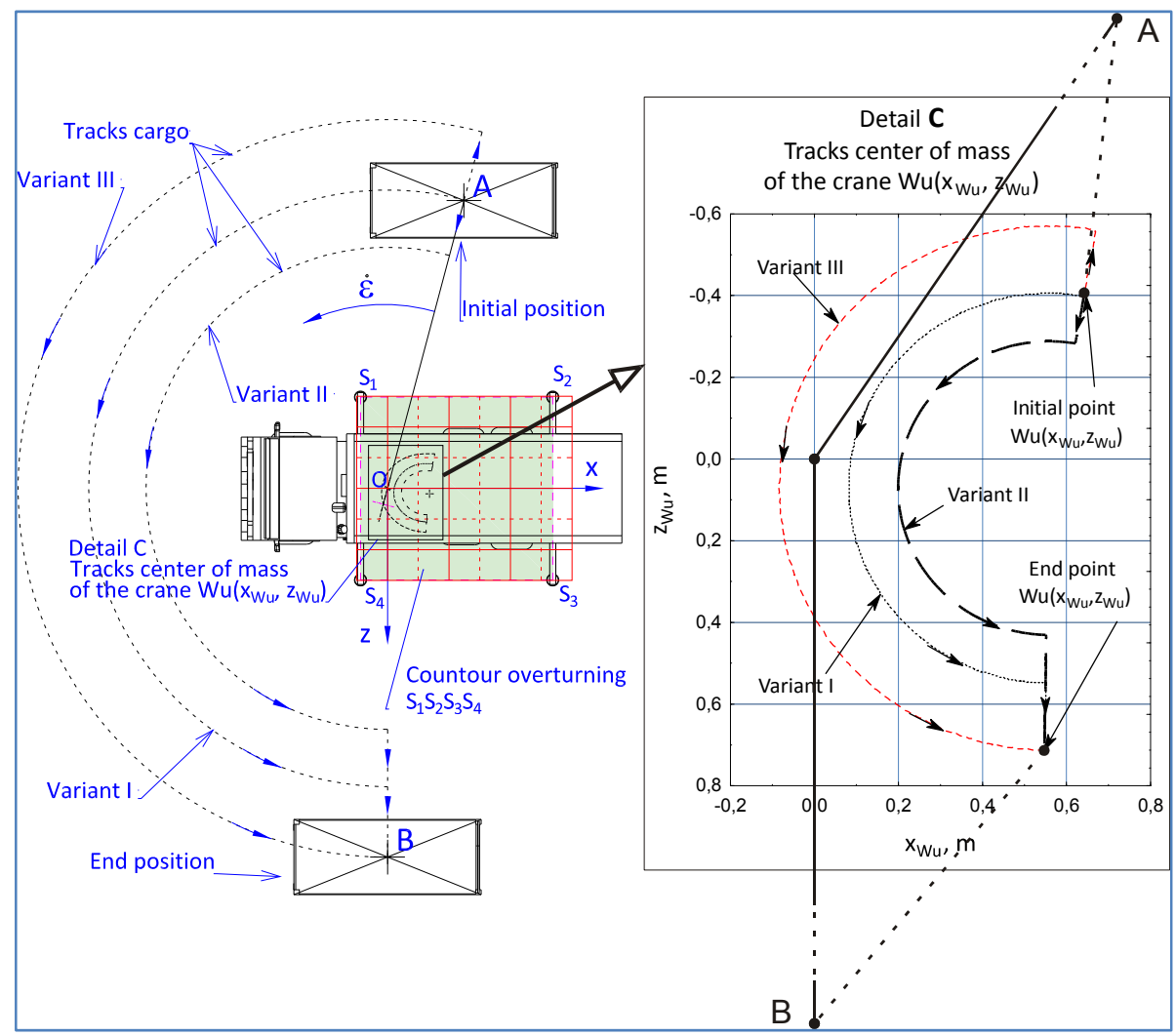

Fig.5. Handling assignment consisting in carrying the cargo from its initial position $A$ to position in point $B$, for three displacement variants.

The integrated CAD/CAE system with an additional computational application was used in simulation testing, which permitted the following among others:

- an accurate determination of the coordinates of any point of the crane system based on the mathematical model that describes its configuration [3];

- establishing the trajectory of the gravity centre of the crane $W u\left(x_{W u}, z_{W u}\right)$,

- calculation of the reaction in the outriggers $R y_{1}, R y_{2}, R y_{3}, R y_{4}=f\left\{G l, W u\left(x_{W u}, z_{W u}\right), t\right\}$ - [19];

- calculation of the difference of the torques $\Delta M=M_{u}-M_{w}=f\left\{G l, W u\left(x_{W u}, z_{W u}\right), t\right\}$;

- calculation of the safety indicator $W b=f\left\{G l, W u\left(x_{W u}, z_{W u}\right), t\right\}$;

- determination of the values of the working loads and the crane lift curves;

- determination of the crane stability conditions as a function of its working load and extension;

- evaluation of the stability of the performance of the entire crane handling cycle;

- optimization of the movement trajectory of the crane working elements for the accepted optimization criterion $W b^{\max }$.

The trajectories presented in Fig. 5 that are determined by the gravity centres $W u\left(x_{W u}, z_{W u}\right)$ of the crane system are located inside the tip-over outline $S_{1} S_{2} S_{3} S_{4}$ (detail C); hence, stability conditions are met. This is confirmed by the changes in the value of the safety indicator $W b$ that are presented in Fig.6. It is evident for the handling assignment example presented that the minimum value of the safety indicator for all of the three cases is greater than 0 ; hence, the crane system is stable over the whole range. For the third 
variant of the handling assignment, however, the value of this indicator $W b=0.002$ is very small. This means that for the trajectory of the load carried $\tau_{H}$ the working conditions are the least favourable as there is a risk of a loss of the crane system stability. This is confirmed by the diagrams in Fig.7 and Fig.8.

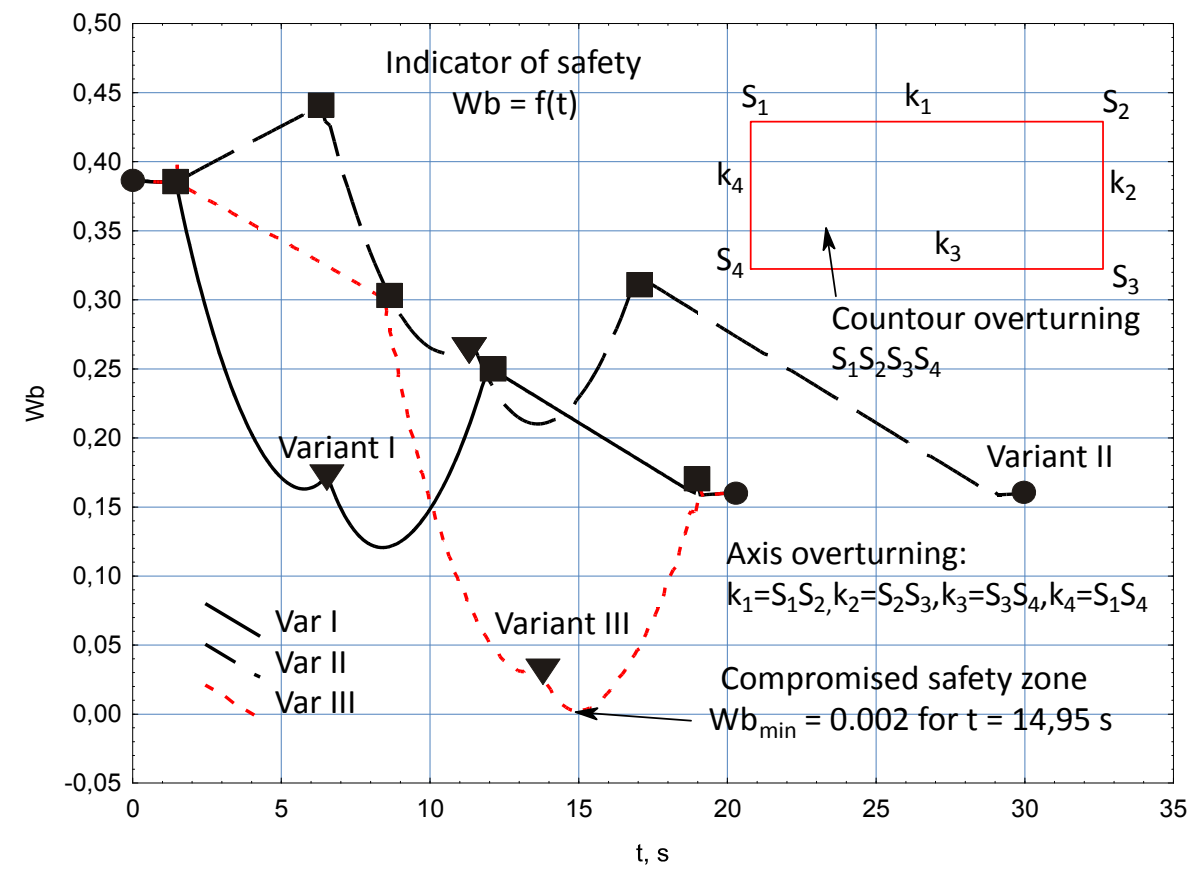

Fig.6. Changes to the value of the safety indicator $W b$ for the three variants of the handling assignment, where: $\bullet$ - start and end of movement, $\square$ - start and end of circular motion, $\nabla$ - change of the tipover axis from $k_{1}$ to $k_{4}$.

a)

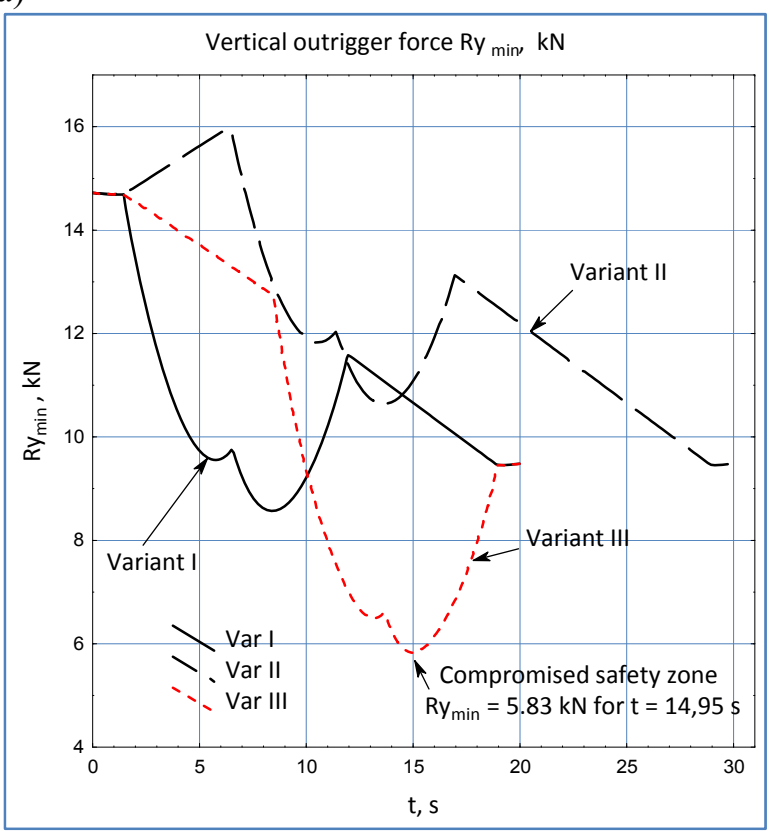

b)

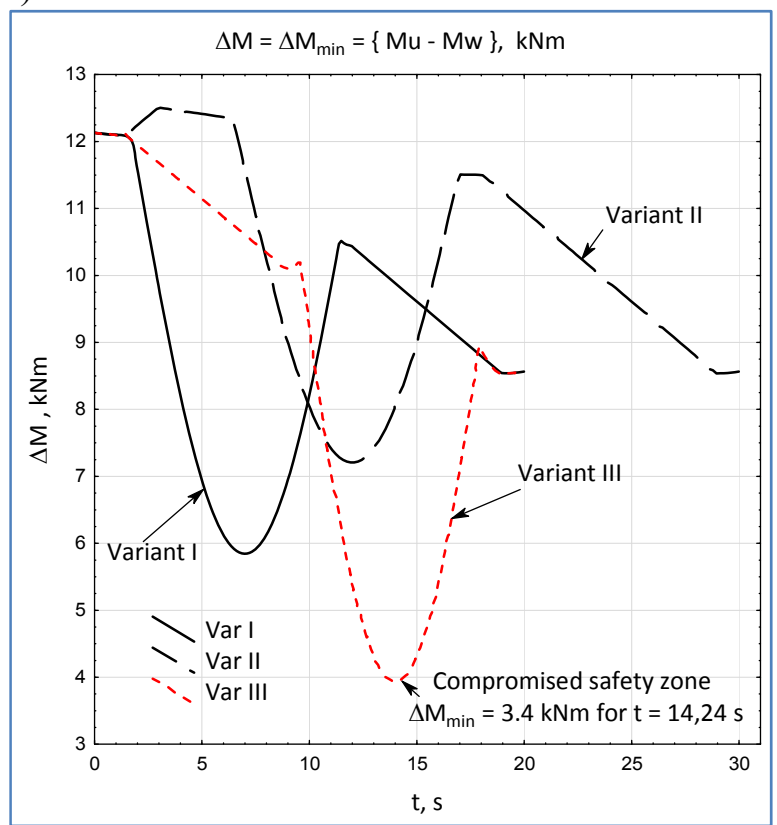

Fig.7. Changes to the value of the base vertical reaction forces $\min \left(R y_{i}\right)$ (a) and values $\Delta M_{\min }$ (b) during displacement of the cargo for the three variants of handling assignment. 
By analysing the changes presented it can be found that in spite of ensuring the crane's static stability, there may occur a risk to its operation (Fig.7: variant III). In the time interval between the $14^{\text {th }}$ and $16^{\text {th }}$ second, the values of the horizontal reaction force $R y_{\min }$ (Fig.7a) and the torque differences $\Delta M$ (Fig.7b) are the lowest. The gravity centre $W u$ (Fig.5) is located too close to the tip-over axis $S_{1} S_{4}$ : as little as in the distance of $d=0.34 \mathrm{~m}$. This is also confirmed by the diagrams in Fig. 8 for the third case of the handling assignment. The values of the vertical reactions $R y_{2}$ and $R y_{3}$ and the torque difference $\Delta M$ are the lowest for this variant.

a)

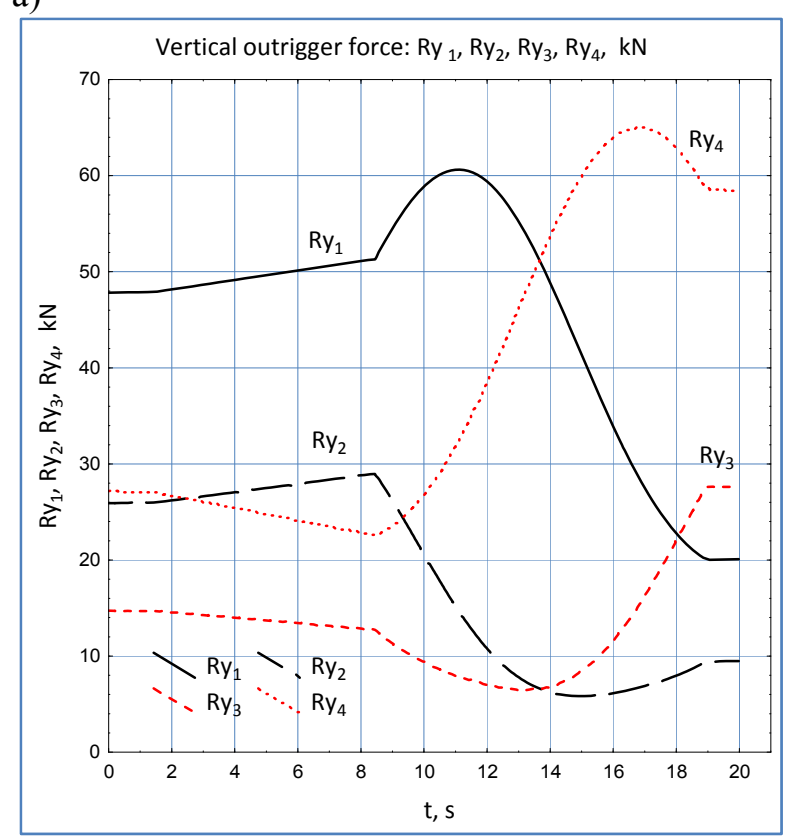

b)

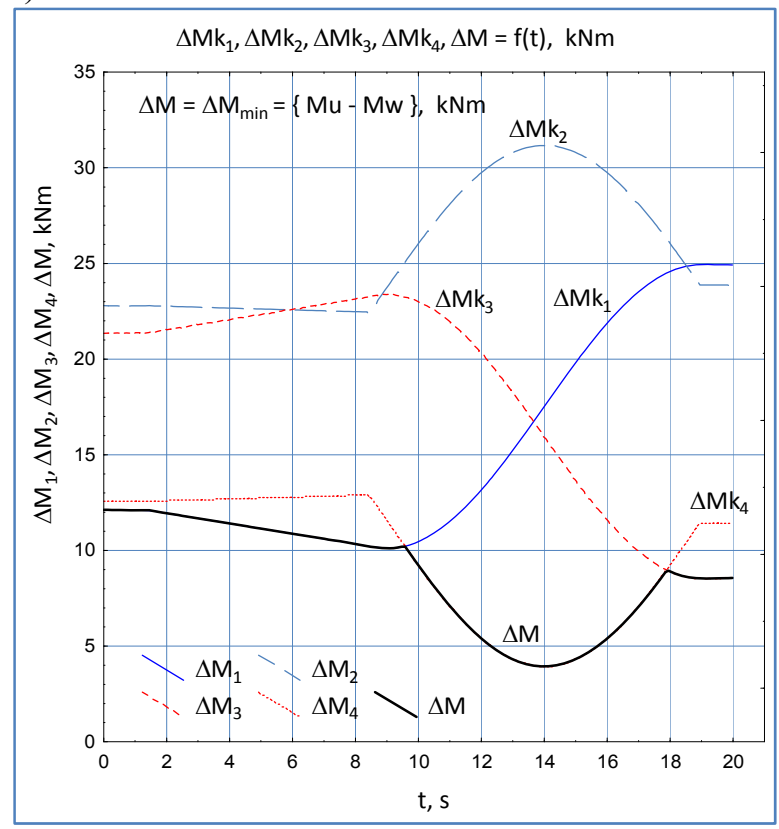

Fig.8. Courses of changes to the values of the vertical reactions of the base $R y_{i}(i=1, \ldots, 4)$ for all the outriggers (a) and the difference of torques $\Delta M_{k}(k=1,2, . ., 4)$ (b) in relation to all the tip-over axes of the crane outrigger system for the third variant of the handling cargo.

The safety indicator $W b$ developed can be useful in an optimization of the trajectory of the handling assignment under execution. In optimization procedures, a maximisation of the indicator $W b$ can be accepted as the objective function when the limiting conditions concern the assignment execution time or the path length of the cargo transported. In another case, when the minimisation of the handling time or path is the objective function, the value of the indicator $W b$ can be a limiting condition.

\section{Conclusions}

The article presents a new approach to the problem of an assessment of the effectiveness of the crane handling system based on the values of the safety indicator for various load conditions and different cargo displacement trajectories with the use of modern $\mathrm{CAD} / \mathrm{CAE}$ computing techniques. The methodology presented in the paper on the example of a mobile crane of the HDS HIAB XS111 type permits an analysis of the movement of the cargo transported for selected configurations.

Owing to the results of numerical simulation that meet stability conditions, it is possible to determine the optimum trajectory of the cargo displacements for the selected handling assignment. The use of the corrections of the displacements of the moving elements of the crane may prevent the outriggers from being broken off, and hence it allows fully safe operation in any conditions. 
The following changes were presented as the results of simulation testing: the location of the gravity centre of the crane system, the reaction of the outrigger system, stabilizing and overturning torques that act on the crane and the value of the safety indicator for the given movement trajectories of the crane working elements.

\section{Acknowledgmentt}

This project is financed by the National Centre for Research and Development, Poland (NCBiR), under the Applied Research Programme - Grant agreement No. PBS3/A6/28/2015.

\section{Nomenclature}

$a, b$ - spacing of the crane outriggers, $[\mathrm{m}]$

$a, b$ - spacing of the crane outriggers, $[\mathrm{m}]$

$d i_{j}$ - distance of the gravity centre of the element $j$ from the tip-over axis $i$ in the projection on the horizontal plane, $[\mathrm{m}]$, Eq.(1.4)

$G_{b}-$ crane base weight, $[N]$

$G_{f}$ - weight of the truck including the outrigger system, $[N]$

$G_{h}$ - hook weight, $[N]$

$G_{k}$ - weight of the slewing column, [N]

$G_{l}$ - cargo weight, $[N]$

$G m_{1}$ - weight of the hydraulic cylinder driving the inner arm, $[N]$

$G m_{2}$ - weight of the hydraulic cylinder driving the outer arm, $[N]$

$G t_{1}, G t_{2}, \ldots G t_{6}$ - weights of the arms of the six-member crane boom, $[N]$

$G_{u}$ - total weight of the crane system, $[k N]$, Eq.(5.1)

$G w_{w}$ - weight of the inner arm, $[N]$

$G w_{z}$ - weight of the outer arm, $[N]$

$k_{1}$ - index of the maximum load of the crane outrigger, Eq.(5.1)

$k_{2}$ - index that determines the minimum load of the crane outrigger, Eq.(5.1)

$M_{u}$ - stabilizing torque, $[\mathrm{kNm}]$, Eq.(1.1)

$M_{w}$ - overturning torque, $[\mathrm{kNm}]$, Eq.(1.1)

$R y_{i}$ - vertical reactions of the base on the outrigger $i,[k N]$, Eq.(5.1)

$t$ - time of the working cycle of the handling assignment, [s], Eq.(5.2)

$t_{b}$ - time of the working cycle of the handling assignment, [s], Eq.(5.2)

$t_{e}-$ end of the crane working cycle, [s], Eq.(5.2)

$W b$ - safety indicator, Eq.(5.1)

$\alpha-$ angle of rotation of the inner arm, $[\mathrm{deg}]$

$\dot{\alpha}-$ rotational velocity of the inner arm, $[\mathrm{deg} / \mathrm{s}]$

$\beta$ - angle of rotation of the outer arm, $[\mathrm{deg}]$

$\dot{\beta}$ - rotational velocity of the outer arm, $[\mathrm{deg} / \mathrm{s}]$

$\Delta M$ - difference of moments, $[\mathrm{kNm}]$, Eq.(1.1)

$\delta t$ - linear displacement of the telescopic arm, $[\mathrm{m}]$

$\dot{\delta} t-$ linear velocity of the six-member telescopic boom, $[\mathrm{m} / \mathrm{s}]$

$\varepsilon$ - angle of rotation of the crane column, $[\mathrm{deg}]$

$\dot{\varepsilon} \quad$ - velocity rotating column, $[\mathrm{deg} / \mathrm{s}]$

$\tau_{H}-$ hook trajectory

$\tau_{L}-$ trajectory of cargo gravity centre

$\cup_{w}$ - the wind speed, $[\mathrm{m} / \mathrm{s}]$

\section{Subscripts}

$b-$ indices for the initial positions

$e-$ indices for the end positions

$i$ - number of the outrigger, (for $i=1,2, \ldots, 4$ )

$j$-number of the elementary fragment of the trajectory, $($ for $j=1,2, \ldots)$ 


\section{References}

[1] Anezirisa O.N, Papazoglou I.A. and Mud M.L. (2008): Towards risk assessment for crane activities. - Safety Science, vol.46, No.6, pp.872-884.

[2] Janusz J. and Klosiński J. (2010): Influence of the selected control strategies of mobile crane motions on its stability. - Acta Mechanica et Automatica, vol.10, No.2, pp.74-80.

[3] Kacalak W., Budniak Z. and Majewski M. (2016): Crane stability for various load conditions and trajectories of load translocation. - Mechanic, No.12, pp. 1820-1823.

[4] Klosinski J. and Janusz J. (2009): Control of operational motions of a mobile crane under a threat of loss of stability. - Solid State Phenomena, vol.144, pp.77-82.

[5] Lei Z., Taghaddos H., Han S., Bouferguene A., Al-Hussein M. and Hermann U. (2015): From AutoCAD to 3ds Max: An automated approach for animating heavy lifting studies. - Canadian Journal of Civil Engineering, vol.42, No.3, pp.190-198.

[6] Posiadala B., Warys P., Cekus D. and Tomala M. (2013): The dynamics of the forest crane during the load carrying. - International Journal of Structural Stability and Dynamics, vol.13, No.7, pp.1340013.

[7] Posiadala B. and Warys.P. (2011): Modeling and simulation research of forest crane in operating cycle. Modelling in Engineering, vol.10, No.41, pp.331-338.

[8] Rauch A., Singhose W., Fujioka D. and Jones T. (2013): Tip-over stability analysis of mobile boom cranes with swinging payloads. - ASME, Journal of Dynamic Systems Measurement and Control, vol.135, No.3:031008, pp.1-6.

[9] Sochacki W. (2007): The dynamic stability of a laboratory model of a truck crane. - Thin-Walled Structures, vol.45, No.10-11, pp.927-930.

[10] Urbaś A. (2013): Analysis of flexibility of the support and its influence on dynamics of the grab crane. - Latin American Journal of Solids and Structures, vol.10, No.1, pp.109-121.

[11] Jeng SL., Yang CF. and Chieng WC. (2010): Outrigger force measure for mobile crane safety based on linear programming optimization. - Mechanics Based Design of Structures and Machines, vol.38, No.2, pp.145-170.

[12] Suwaj S. and Mączyński A. (2002): Stability inspection of a crane during working movements. -Industrial transport, No.4/10, pp. 26-29.

[13] Arena A., Casalotti A., Lacarbonara W. and Cartmell M.P. (2015): Dynamics of container cranes: threedimensional modeling, full-scale experiments, and identification. - International Journal of Mechanical Sciences, vol.93, pp.8-21.

[14] Lee J.S.W., Shim, J.J., Han D.S., Han G.J. and Lee K.S. (2007): An experimental analysis of the effect of wind load on the stability of a container crane. - Journal of Mechanical Science and Technology, vol.21, No.3, pp.448454.

[15] Lee J.S. and Kang J.H. (2008): Wind load on a container crane located in atmospheric boundary layers. - Journal of Wind Engineering and Industrial, vol.96, No.2, pp.193-208.

[16] ISO 4305:2014. - Mobile cranes - Determination of stability.

[17] PN-ISO 4304:1998. - Tower cranes, Stability requirements.

[18] PN-ISO 4305:1998. - Mobile cranes - Determination of stability.

[19] Kacalak W., Budniak Z. and Majewski M. (2018): Computer aided analysis of the mobile crane handling system using computational intelligence methods. - Advances in Intelligent Systems and Computing, vol.662, pp.250261.

[20] Rupar D., Hladnik J. and Jerman B. (2016): Loader crane inertial forces. - FME Transactions, vol.44, No.3, pp.291-297. 
[21] Majewski M. and Kacalak W. (2017): Innovative intelligent interaction systems of loader cranes and their human operators. - Advances in Intelligent Systems and Computing, vol.573, pp.474-485.

[22] Majewski M., Kacalak W., Budniak Z. and Pajor M. (2018): Interactive control systems for mobile cranes. Advances in Intelligent Systems and Computing, vol.661, pp.10-19.

[23] Herbin P. and Pajor M. (2016): Modeling direct and inverse kinematics of loading crane with redundant degrees of freedom structure using Matlab. - Modelling in Engineering, vol.27, No.58, pp.44-50.

[24] Kacalak W., Budniak Z. and Majewski M. (2016): Simulation model of a mobile crane with ensuring its stability. - Modelling in Engineering, vol.29, No.60, pp.35-43.

Received: August 21, 2017

Revised: December 4, 2017 